\title{
Aberration-Corrected STEM for Understanding of the Catalytic Mechanisms and Development of New Catalysts
}

\author{
Albina Y. Borisevich*, Andrew R. Lupini*, Sergey N. Rashkeev**,*, Sanwu Wang**, Gabriel M. \\ Veith*, Nancy J. Dudney*, S.T. Pantelides**,*, Stephen J. Pennycook*,** \\ *Condensed Matter Sciences Division, Oak Ridge National Laboratory, Oak Ridge, TN 37831, USA \\ **Dept. Physics and Astronomy, Vanderbilt University, Nashville, TN 37235, USA
}

Aberration-corrected Z-contrast (HAADF) STEM has proven an invaluable tool for catalysis research due to its superior resolution and high sensitivity. ORNL's 300 kV VG Microscopes' HB603U STEM with Nion aberration corrector was shown to resolve lattice spacings as small as $0.78 \AA[1]$ and routinely allows us to image single atoms inside the materials and on their surfaces as well. The ability to simultaneously collect EELS spectra provides additional possibilities for characterization. All these aspects have proven instrumental for the studies of catalytic activity and degradation in Cr-doped transition aluminas, which are widely used for dehydrogenation of alkanes [2]. Even though structural difference between $\gamma-\mathrm{Al}_{2} \mathrm{O}_{3}$ and $\eta-\mathrm{Al}_{2} \mathrm{O}_{3}$ is minimal and only amounts to different distribution of cation vacancies within spinel framework, Cr-doped $\eta-\mathrm{Al}_{2} \mathrm{O}_{3}$ catalysts can last several years, while $\mathrm{Cr} / \gamma-\mathrm{Al}_{2} \mathrm{O}_{3}$ catalysts degrade in weeks [3]. Our STEM studies revealed that $\mathrm{Cr}$ dopant is distributed differently in these two systems. On $\gamma-\mathrm{Al}_{2} \mathrm{O}_{3}, \mathrm{Cr}$ is segregated into extended "patches" of $\mathrm{Cr}_{2} \mathrm{O}_{3}$ (Fig. 1). On $\eta-\mathrm{Al}_{2} \mathrm{O}_{3}$, on the other hand, no segregation was observed. Contrast in the STEM images was mostly uniform, with the thinnest areas of the alumina flakes sometimes displaying faint spots (Fig.2a)). The spots have similar intensity (Figs. 2b)-d)) of about the right level for Cr. EELS studies (Fig. 2e)) also indicate uniform distribution of dopant on $\eta-\mathrm{Al}_{2} \mathrm{O}_{3}$ surface. Our first-principles calculations suggest that catalytic processes primarily happen on isolated $\mathrm{CrO}_{\mathrm{x}}$ species, and preserving a high density of these active sites is necessary to retain activity. Due to a unique surface reconstruction resulting from its specific distribution of cation vacancies, $\gamma-\mathrm{Al}_{2} \mathrm{O}_{3}$ (110C) surface can accommodate growth of extended $\mathrm{Cr}_{2} \mathrm{O}_{3}$ "patches', while on $\eta-\mathrm{Al}_{2} \mathrm{O}_{3} \mathrm{Cr}$ remains isolated, resulting in much slower degradation.

STEM can also provide valuable guidance in development of new catalysts. For example, materials obtained via magnetron sputtering of metal onto oxide powder [4] when studied by STEM demonstrate highly dispersed and uniform metal distribution (Fig. 3). Adhesion can be achieved in systems not accessible by chemical routes, such as $\mathrm{Au} / \mathrm{SiO}_{2}$. The method also shows promise for non-oxide substrates such as ionic liquids or metals and can potentially be used for synthesis of alloy nanoparticles. STEM can also be very useful for development of novel bimetallic catalysts, where we can assess the distribution of the different metals on the substrate and sometimes trace the evolution of the metal clusters starting from the original molecules (Fig. 4). New developments in 3D STEM of catalysts will also be discussed (see more in [5]) [6].

References

[1] P. D. Nellist et al., Science 305 (2004) 1741.

[2] C. N. Satterfield, Heterogeneous Catalysis in Practice, McGraw Hill, New York, 1980.

[3] R. Iezzi et al. U.S. Patent No. 6,362,385 (March 26, 2002).

[4] G. M. Veith et al., J. Catal., in press (2005).

[5] A. R. Lupini et al., these proceedings

[6] We are grateful to Prof. R. D. Adams for the provision of bimetallic catalyst samples. This work was supported by USDOE under contract DE-AC05-00OR22725 managed by UT-Battelle, LLC 

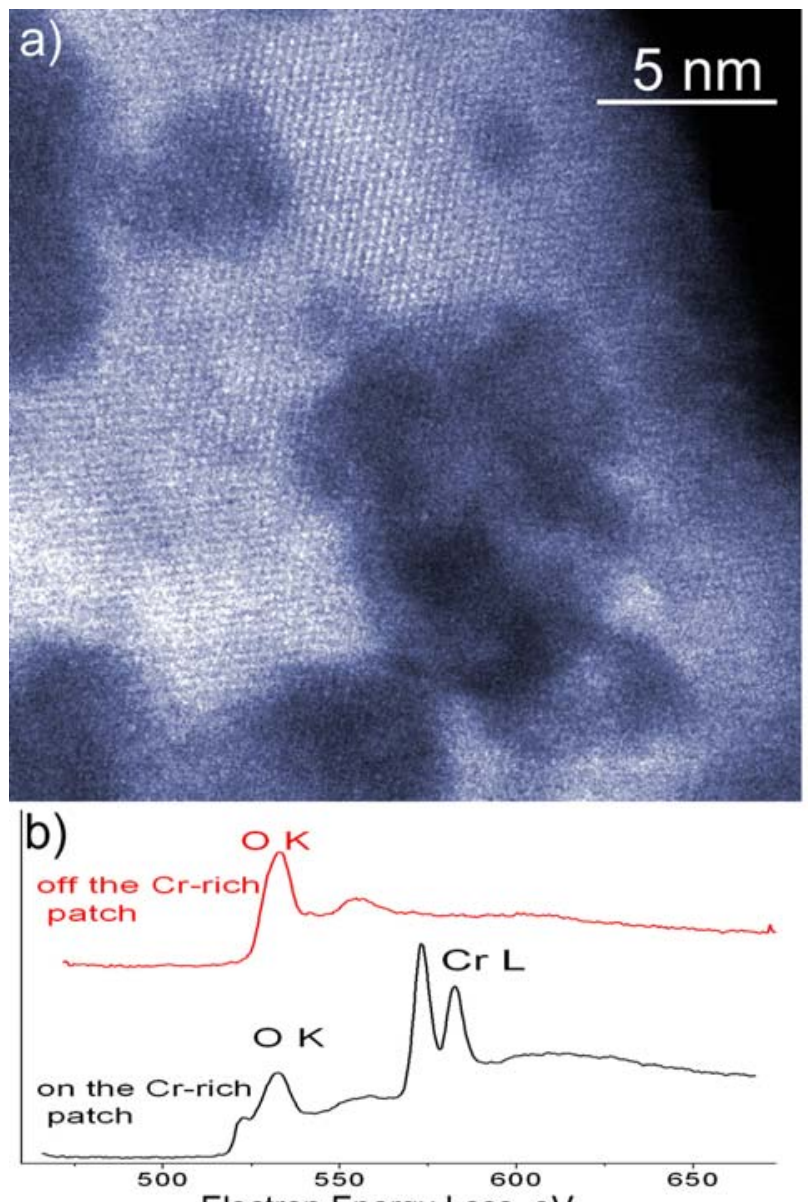

Fig.1. Cr-doped $\gamma-\mathrm{Al}_{2} \mathrm{O}_{3}$. a) STEM image (smoothed) of a $\gamma-\mathrm{Al}_{2} \mathrm{O}_{3}$ flake with $\mathrm{Cr}_{2} \mathrm{O}_{3}$ patches; b) EELS spectra collected on the patches and between them, confirming $\mathrm{Cr}_{2} \mathrm{O}_{3}$ segregation.

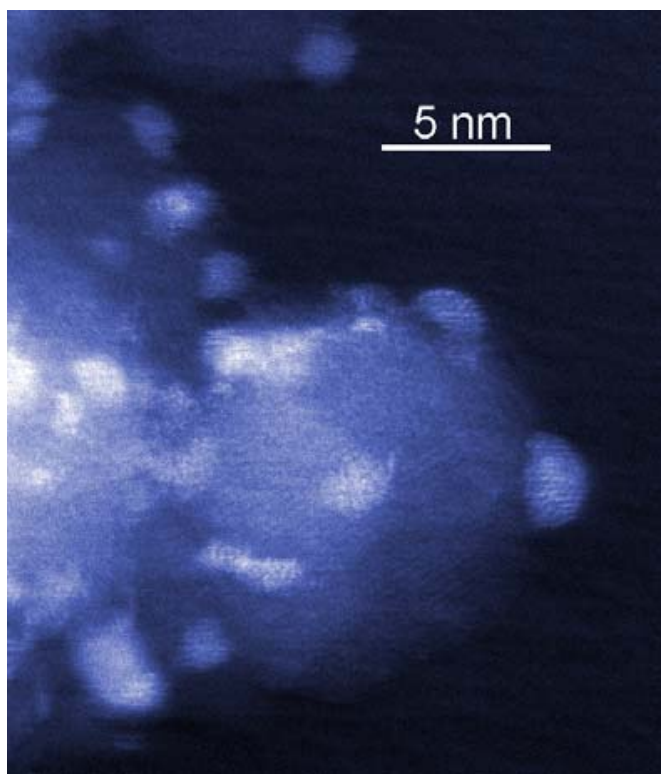

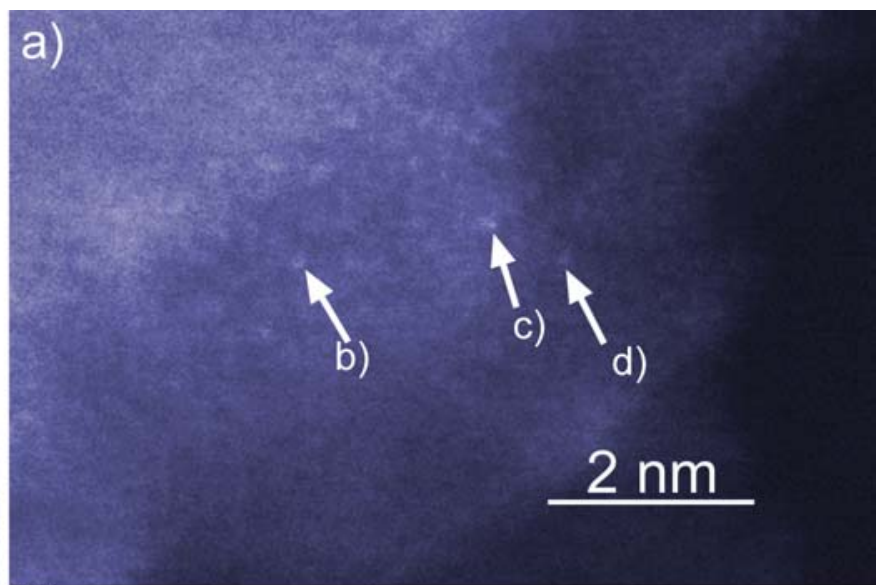
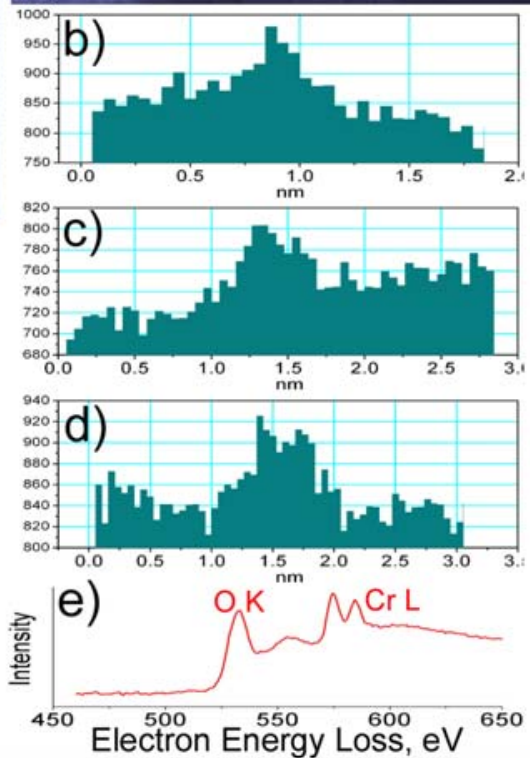

Fig.2. Cr-doped $\eta-\mathrm{Al}_{2} \mathrm{O}_{3}$. STEM image (smoothed) of an $\eta-\mathrm{Al}_{2} \mathrm{O}_{3} \quad$ flake with possible single $\mathrm{Cr}$ atoms arrowed; b), c) and d) corresponding profiles; e) EELS spectrum confirming the presence of $\mathrm{Cr}$.
Fig. 3.

STEM image (raw data) of the $\mathrm{Au} / \gamma-\mathrm{Al}_{2} \mathrm{O}_{3}$ catalyst obtained via magnetron sputtering (see text)

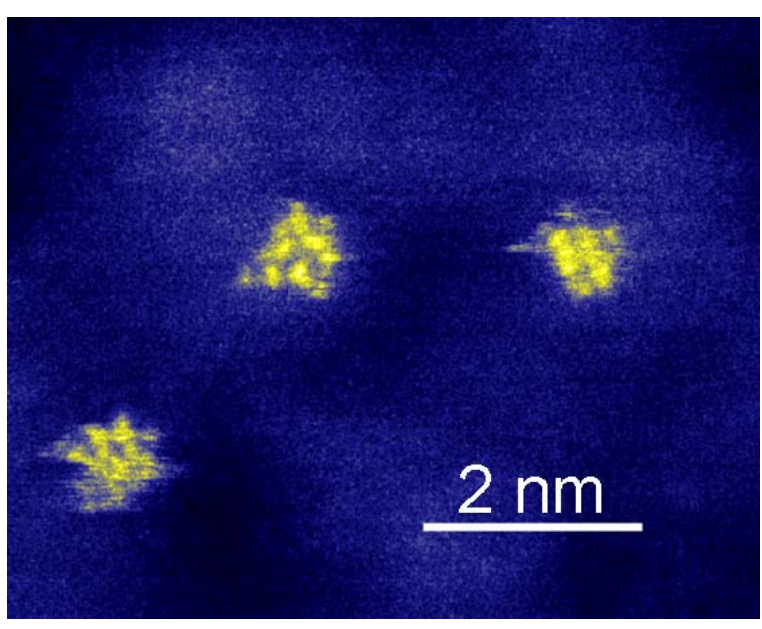

Fig. 4. STEM image (smoothed) of the $\mathrm{Ir}_{8} \mathrm{Pt}_{4}$ clusters on $\mathrm{MgO}$ surface - intact metal cores of the $\mathrm{Ir}_{8} \mathrm{Pt}_{4}\left(\mathrm{PBu}_{3}^{\mathrm{t}}\right)_{4}(\mathrm{CO})_{12}$ molecules. 